\title{
Psicooncología
}

ISSN: 1696-7240

\section{Comunicación consciente para acompañar de forma eficaz: protocolo de 5 pasos}

\author{
Sophia Denizon Arranz ${ }^{1 *}$; Pilar Arranz Carrillo de Albornoz ${ }^{2}$; Cristina Coca Pereira ${ }^{3}$; Jesús \\ Manuel Pérez Viejo ${ }^{4}$; Miguel Costa Cabanillas ${ }^{5}$; Emilio Cervera Barba ${ }^{6}$; Helena García Llana
}

Recibido: 9 de noviembre de 2019 / Aceptado: 20 de enero de 2020

Resumen: Las personas con enfermedades crónicas y graves, necesitan ser escuchadas, entendidas y atendidas a su ritmo. Los profesionales necesitan herramientas sencillas y eficientes que les faciliten una buena comunicación con sus pacientes, en el menor tiempo posible. Ojetivo: describir un protocolo sistemático de comunicación en cinco pasos. Método: Se ha diseñado un enfoque cualitativo basado en la revisión documental, que ha incluido la revisión bibliográfica y de evidencias para apoyar el análisis y poder llegar a conclusiones con la información extraída del proceso. Resultado: Como resultado se propone un protocolo básico de comunicación fundamentado en el counselling y en los principios de la bioética moderna, capaz de facilitar las funciones de informar, motivar la adhesión terapéutica, promover cambios de conducta, abordar situaciones de comunicación difícil y, especialmente, de tener un encuentro auténtico con el paciente y su familia. Por conclusión sin comunicación eficaz no hay encuentro, y sin encuentro, la relación clínica entre profesional y paciente se puede convertir en un intercambio de palabras vacías, de imposiciones de tratamientos, que difícilmente el paciente va a cumplir adecuadamente. Los tiempos cambian y la Medicina participativa va encontrando un lugar más adecuado en el entorno clínico.

Palabras clave: Competencias relacionales en sanitarios; protocolo comunicación; counselling; relaciones profesionales sanitarios-paciente; medicina participativa.

1 Sophia Denizon Arranz, Centro de Simulación Clínica de la Universidad Francisco de Vitoria. Facultad de Medicina. Madrid, España.

E-mail: sophia.denizon@ufv.es

2 Pilar Arranz Carrillo de Albornoz, Servicio de Hematología Hospital Universitario La Paz (1974-2005) Socia Fundadora Instituto Antae: Salud y Personas. Madrid, España.

E-mail: piarranzpaz@gmail.com

3 Cristina Coca Pereira Universidad Europea de Madrid, Valencia International University, Universidad Autónoma de Madrid. Madrid, España.

E-mail: cristina.coca@yahoo.es

4 Jesús Manuel Pérez Viejo, Universidad Internacional de la Rioja. Madrid, España.

E-mail: jesusmanuel.perez@unir.net

5 Miguel Costa Cabanillas, Facultad de Psicología. Universidad Autónoma de Madrid. Madrid, España.

E-mail: mcosta@cop.es

6 Emilio Cervera Barba, Facultad de Medicina. Universidad Francisco de Vitoria. Madrid, España.

E-mail: emilio.cervera@ufv.es

7 Helena García Llana, Hospital Universitario La Paz-IdiPAZ. Profesora Asociada. Departamento de Psicología. Universidad Pontificia de Comillas. Profesora Asociada. División de Psicología. Centro de Enseñanza Superior Cardenal Cisneros adscrito a la Universidad Complutense de Madrid. Madrid, España.

E-mail: helenagllana@hotmail.com

* Dirección de correspondencia: Sophia Denizon Arranz. Centro de Simulación Clínica de la Universidad Francisco de Vitoria. Facultad de Medicina. Carr. de Pozuelo de Alarcón, 800, Km 1, 28223 Majadahonda, Madrid. E-mail: sophia.denizon@ufv.es 


\title{
[en] Conscious communication to accompany effectively: protocol of 5 steps
}

\begin{abstract}
People with chronic and serious diseases, need to be heard, understood and answered at their own pace. Professionals need simple and efficient tools to have good communication with their patients, in the shortest possible time. The aim of this paper is to describe a systematic communication in five steps, protocol based on counselling and on the principles of bioethics, to facilitate the information skills, motivate therapeutic adherence, promote behavioral changes, deal with difficult situations and to have an authentic encounter with the patient and his family. It is concluded that without effective communication there is no encounter, and without encounter, the clinical relationship between professional and patient can become an exchange of empty words, of impositions of treatments, which the patient will hardly adequately comply with. Times change and participatory medicine is finding a more appropriate place in the clinical environment.

Keywords: Relational competences in health care; communication protocol; counselling; healthcare professional-patient relationships; participatory medicine.
\end{abstract}

Sumario: 1. Introducción 2. Antecedentes: Counselling y Medicina Participativa 3. Escalera de comunicación en 5 pasos (PCBC-S): Cuando menos es más.

Cómo citar: Denizon Arranz S, Arranz Carrillo de Albornoz P, Coca Pereira C, Pérez Viejo JM, Costa Cabanillas M, Cervera Barba E, García Llana H. Comunicación consciente para acompañar de forma eficaz: protocolo de 5 pasos. Psicooncología 2020;17:165-177. doi: 10.5209/psic.68248.

\section{Introducción}

Las habilidades relacionales son funciones prioritarias de los profesionales sanitarios, si bien en múltiples ocasiones se dan por sabidas, por lo que se les confiere poca importancia ${ }^{(1)}$. En 1991, la declaración de Toronto ${ }^{(2)}$ ya se hacía eco de los importantes problemas de la práctica sanitaria en sus relaciones con los pacientes. Se prescriben diferentes tratamientos, con frecuencia complicados y con efectos secundarios; se dan consejos del tipo: realizar determinada dieta, hacer ejercicio físico, tomarse las cosas con tranquilidad, etc. Algunos profesionales se desesperan al comprobar la falta de adhesión a los tratamientos y que sus bienintencionados consejos no obtienen los resultados que esperaban ${ }^{(3)}$.

¿Por qué conforman un área competencial particularmente importante? a) para recoger los datos clínicos necesarios que faciliten una comprensión de lo que le sucede al paciente y así llegar a un diagnóstico integral y personalizado; b) para promover comportamientos saludables, que prevengan enfermedades, favorezcan su control y disminuyan sus complicaciones; c) para informar adecuadamente sobre diagnósticos y tratamientos; d) para llegar a acuerdos en la toma de decisiones. Por tanto, es fundamental formar en habilidades de comunicación de un modo efectivo y gratificante tanto para los profesionales como para los pacientes.

Como defiende Ruíz Moral ${ }^{(4)}$, estas habilidades representan un componente esencial de las competencias en el ámbito sanitario. La literatura describe la influencia beneficiosa de la comunicación sobre distintos aspectos del ámbito clínico $^{(5)}$, resumidos en la Tabla 1 . Además, contribuyen a mejorar la expectativa de vida, predicen un mayor bienestar y una mejor salud ${ }^{(6)}$. 
Tabla 1. Importancia de la comunicación en el ámbito clínico

1. La comunicación permite realizar diagnósticos más precisos.

2. Fomenta la adhesión al tratamiento.

3. Una buena relación entre médico-paciente es, en sí misma, terapéutica, genera expectativas positivas e influye en los resultados clínicos al activar mecanismos biológicos y redes neuronales implicadas en el efecto placebo.

4. Aumenta la influencia para introducir cambios de hábitos en la vida de los pacientes.

5. Mejora la eficiencia de la gestión del tiempo.

6. Disminuye el número de demandas en su contra.

7. Reduce síntomas de un modo significativo.

8. Aumenta el grado de satisfacción tanto del profesional como de los pacientes y familias.

9. Disminuye el burnout del profesional.

10. Mejorar el clima social del equipo y su efectividad.

Tomado de: Costa y Arranz ${ }^{(5)}$.

Por otro lado, sabemos que el éxito del tratamiento se debe, en parte, al efecto de la relación de confianza y a las expectativas positivas del paciente ${ }^{(7-9)}$.

Se da la paradoja de que, existiendo una consolidada base empírica que sostiene la importancia de las habilidades relacionales en el perfil de los médicos, enfermeras, psicólogos y trabajadores sociales, sea ésta tan difícil de incorporar en los itinerarios formativos del currículum de formación. Una de las justificaciones es que se trata de un tema muy complejo. La comunicación humana es, a escala del comportamiento, el elemento más complejo ${ }^{(10)}$.

Dicha complejidad se ha incrementado con el nuevo paradigma relacional en que la atención clínica se centra en las personas, transformándose el modelo paternalista hacia un modelo de medicina participativa, deliberativa y personalizada. La sociedad -y los pacientes oncológicos y sus familias como miembros activos de éstaesperan respeto a sus derechos y valores. Para ello se requiere desarrollar actitudes y habilidades de comunicación en la relación clínica, que permitan sintonizar, informar adecuadamente y obtener el consentimiento del paciente, promoviendo su participación en la toma de decisiones ${ }^{(11)}$.

Como consecuencia, se les exige a los profesionales sanitarios una mayor proactividad y un importante esfuerzo. Deberán asumir un mayor compromiso en la resolución de problemas complejos, conocer sus reacciones emocionales y aprender a gestionarlas; escuchar, entender $\mathrm{y}$ atender las necesidades, preocupaciones y reacciones emocionales de los pacientes y sus familias; reflexionar antes de actuar, teniendo en cuenta las preferencias de los pacientes y su historia de valores para llegar a acuerdos, etc.

¿Cómo formar en habilidades de comunicación y hacer que sea coherente y gratificante para todos los componentes del escenario asistencial en oncología?

Para facilitar este proceso formativo está ampliamente demostrado que los métodos de aprendizaje experienciales son más eficaces que otras metodologías convencionales ${ }^{(4,12)}$. Se cuenta con hospitales simulados, la aplicación de juego de 
roles, videos, actores simulados, ejercicios reflexivos en pequeños grupos, portfolios, análisis de escenas de películas, etc. Los objetivos son: despertar la conciencia de la importancia de la comunicación eficaz con los pacientes y fomentar su motivación por aprenderla e integrarla en la práctica clínica diaria.

Es necesario y oportuno para facilitar dicho aprendizaje, proveer de herramientas que ayuden a sistematizar el proceso comunicativo. Con este fin, se propone un protocolo de comunicación básico adaptado a la oncología que optimice el encuentro profesional sanitario-paciente, estructurado en cinco pasos. Los objetivos son los siguientes: a) proponer un instrumento en el contexto del nuevo paradigma de la Medicina Participativa; b) integrar los aspectos clínicos con los relacionales en el escenario asistencial actual respetando la Ley 41/2002 básica reguladora de la de Autonomía del paciente y de derechos y obligaciones en materia de información y documentación clínica en su última modificación de $2015^{(13)}$; c) sistematizar los amplios y complejos elementos que se dan en la comunicación para aplicarlos de forma sencilla; d) maximizar el nivel de competencia en las habilidades de comunicación de los profesionales y minimizar, tanto como sea posible, el coste emocional del paciente, allegados y del profesional que les asiste.

Se trata de un protocolo en el que se combina estructura y proceso, con capacidad para afrontar tanto una entrevista clínica como encuentros puntuales con pacientes y/o familiares, o en interacciones delicadas con el equipo de trabajo.

\section{Antecedentes: Counselling y Medicina Participativa}

Desde la primera Carta de los Derechos de los Pacientes en 1973, aprobada por la Asociación Americana de Hospitales ${ }^{(14)}$ y que en 1984 la Organización Mundial de la Salud promoviera una nueva relación con los pacientes que padecían VIH/SIDA fue necesario que los pacientes compartieran la toma de decisiones ${ }^{(15)}$.

Los pacientes requieren distintos estilos comunicativos. La coexistencia de pacientes que demandan estilos más o menos directivos o participativos suele ocasionar dificultades en la intercomunicación. Por otro lado, los valores, creencias y emociones del interlocutor influyen en su comprensión y en la relación entre ambos. Se trataba de buscar las herramientas más eficientes que facilitaran un encuentro autentico entre profesionales y pacientes, y plasmarlas en un protocolo que recogiera los puntos claves para que la información fuera bien recibida por los pacientes y que, al mismo tiempo, al profesional sanitario le resultara sistemática, coherente y efectiva.

Nuestra propuesta se ha fundamentado en el marco teórico del Counselling, herramienta primordial de la Medicina Participativa. Consiste en un proceso deliberativo e interactivo-relacional entre paciente y profesional que fomenta la aceptación de la enfermedad, reduce los estados emocionales adversos, promueve la autorregulación del profesional y favorece la motivación al cambio desde modelos de potenciación y resiliencia ${ }^{(16)}$.

El Counselling se enmarca en una relación de respeto hacia uno mismo y hacia los demás, también en el compromiso ético primordial de no maleficencia y ecuanimidad, promoviendo la beneficencia y la autonomía en la relación interpersonal. Son fundamentales las actitudes que lo sostienen: acogida empática $^{(17)}$; presencia y compasión ${ }^{(18)}$. El Counselling tiene cuatro pilares 
fundamentales: autorregulación, comunicación proactiva, apoyo emocional y toma de decisiones compartida.

La propuesta de este protocolo surge de la experiencia en la práctica clínica interdisciplinar de escucha atenta a las necesidades, preocupaciones y recursos tanto de los pacientes, como de los profesionales con el fin de extraer la esencia que rige el encuentro en situaciones clínicas, donde existe una asimetría técnica -que no moral-.

El proceso de adaptación del Protocolo de Comunicación Básico para profesionales sanitarios de Cinco pasos (PCBC-S) a nuestro contexto sociocultural se realizó a partir de las herramientas desarrolladas en la Guía de Calgary Cambridge para el proceso de comunicación en la entrevista médica ${ }^{(19)}$ y de los trabajos realizados por un equipo interdisciplinar compuesto por médicos, enfermeros y psicólogos del equipo que lideraron Fernando Hernández Navarro y Pilar Arranz en el Servicio de Hematología del Hospital Universitario La Paz de Madrid, durante más de 30 años $^{(20-}$ 29). Se ha difundido en los programas formativos en habilidades de comunicación, de counselling y otro tipo de competencias asociadas, a través de la Escuela Nacional de Sanidad, de Masters de Psicooncología (UCM, UB), de Masters de Cuidados Paliativos (UAM, UAB, UM, UV, ICO, UNIR) y Masters relacionados con la atención a enfermos crónicos (UV, UCM, UPV, URJC, UPCM, UAM), así como en numerosos cursos impartidos en Hospitales públicos y privados, Universidades españolas e internacionales, talleres, proyectos y programas formativos realizados a través del Instituto Antae, llegando a más de 18.000 profesionales de la salud con excelentes resultados cualitativos. El instrumento final, tras el proceso de adaptación, quedó simplificado en cinco pasos que se describen a continuación (ver figura 1).

Figura 1. Protocolo básico de comunicación eficaz en cinco pasos.

\section{Protocolo básico de comunicación eficaz en cinco pasos:}

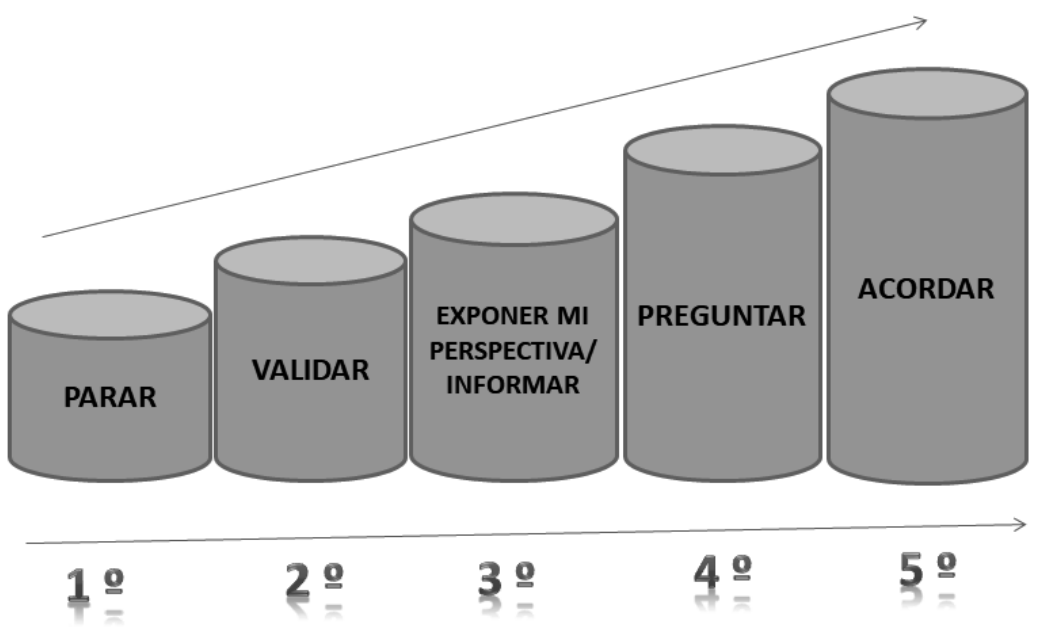

Tomado de: Costa y Arranz 


\section{Escalera de comunicación en 5 pasos (PCBC-S): Cuando menos es más.}

\section{$1^{\circ}$. Preparar el encuentro: Parar para conectarse.}

Atendiéndose para atender en el día a día: Ana es psicooncóloga y tiene que ver a 6 pacientes en el Hospital de Día. Le llaman de la planta informándola sobre unos familiares muy angustiados porque les acaban de dar una mala noticia. "madre mia! Voy fatal de tiempo y siento mucho el dolor de esta familia, quería relajar a Antonio antes de la quimio...pues si no me relajo yo antes, poco podré hacer...tranquila, respira profundo y prioriza..."

El entorno académico y sanitario suele formar a los profesionales en el paradigma biomédico, en el que la realidad es solo lo objetivamente cuantificable. Sin embargo, lo que encontramos en cada consulta es una persona con sus preocupaciones, miedos, dudas, sufrimientos que no siempre se pueden medir, pero, afortunadamente, si se pueden identificar, escuchar, entender y atender. La relación clínica es un encuentro que se rige por la ley de interdependencia donde el propio comportamiento es decisivo en el proceso de comunicación. El profesional sanitario debe hacerse cargo de la dirección de la comunicación en función de los objetivos profesionales. De ahí la importancia de estar atentos, conectados, elegir qué tipo de relación y comunicación es la adecuada en ese momento en particular con ese preciso interlocutor, se trate de pacientes, familiares o compañeros de equipo. En la comunicación eficaz debe primar la coherencia con nuestros objetivos, no la impulsividad automática, reactiva, aprendida y sin conciencia.

¿Cómo parar y responder de un modo proactivo y no dejarse llevar por los automatismos?: Pensar antes de actuar. La comunicación consciente es difícil y requiere entrenamiento. Cuando se logra, es tan gratificante la consecuencia, que compensa el esfuerzo de realizarlo. Responder de forma proactiva requiere un proceso de autorregulación en el que es útil prestar atención a la propia respiración, ya que se produce en el aquí y el ahora, informándonos del propio estado emocional para conectarse en el presente, desde donde poder elegir cómo se quiere actuar.

Asimismo, es importante conectar con las propias emociones, reconocerlas y reconducirlas, antes de pasar a la acción ${ }^{(29)}$. Este proceso resulta de gran utilidad, al disminuir el desgaste asociado al trabajo implicado con el sufrimiento. Permite el autocuidado emocional, dotar de sentido a las acciones clínicas, cooperar mejor con el equipo y disponer de habilidades para afrontar situaciones críticas.

\section{$2^{\circ}$ Preparar la relación: Acoger y Validar para escuchar y comprender.}

La amabilidad y el respeto dejan huella: Olga es psicooncóloga desde hace 25 años. En la planta hospitalaria de oncología atiende a muchos pacientes con distintos diagnósticos oncológicos. Se toma un par de segundos para prepararse para acoger cada paciente.

Ps: Hola Pedro, buenos días. ¿Cómo se encuentra hoy?

Pac: Pensar que vengo al Hospital me enferma

Psi: Lo entiendo, es un proceso largo y duro 
Tabla 2. Tenemos la opción de elegir cómo actuar entre.

\begin{tabular}{|c|c|c|c|}
\hline \multicolumn{2}{|c|}{$\begin{array}{l}\text { ESTILO REACTIVO: } \\
\text { Inhibido o agresivo }\end{array}$} & \multicolumn{2}{|c|}{$\begin{array}{l}\text { o ....ESTILO PROACTIVO: } \\
\text { Asertivo o consciente }\end{array}$} \\
\hline $\begin{array}{l}\text { No está conectado. } \\
\text { Se reacciona } \\
\text { inhibiendo } \\
\text { emociones. }\end{array}$ & $\begin{array}{l}\text { Pensamientos } \\
\text { (Estoy super } \\
\text { enfadado. No pienso } \\
\text { decir nada, que se } \\
\text { fastidie...) }\end{array}$ & $\begin{array}{l}\text { Está conectado con } \\
\text { sus emociones, } \\
\text { las escucha y las } \\
\text { valida. }\end{array}$ & $\begin{array}{l}\text { “Me siento } \\
\text { enfadado, es normal, } \\
\text { con todo lo que he } \\
\text { hecho, el paciente } \\
\text { sigue exigiendo... sus } \\
\text { razones tendrá”. }\end{array}$ \\
\hline $\begin{array}{l}\text { - No está conectado. } \\
\text { Se reacciona con } \\
\text { violencia }\end{array}$ & $\begin{array}{l}\text { "¡Pero de qué } \\
\text { va! Todo el día } \\
\text { exigiendo" }\end{array}$ & $\begin{array}{l}\text { Se para, se conecta } \\
\text { y piensa en sus } \\
\text { objetivos. }\end{array}$ & $\begin{array}{l}\text { ¿Cómo quiero } \\
\text { actuar?" }\end{array}$ \\
\hline $\begin{array}{l}\text { - Impone, no } \\
\text { escucha. }\end{array}$ & $\begin{array}{l}\text { "Lo que tiene que } \\
\text { hacer es exigir } \\
\text { menos y hacer más } \\
\text { caso" }\end{array}$ & $\begin{array}{l}\text { Escucha y pasa a la } \\
\text { acción de manera } \\
\text { asertiva. }\end{array}$ & $\begin{array}{l}\text { "Le veo preocupado } \\
\text { ¿Puedo hacer algo } \\
\text { por usted?" }\end{array}$ \\
\hline
\end{tabular}

Adaptado de Costa y Arranz ${ }^{(5)}$.

Para acoger al paciente en un principio basta, sencillamente, con saludar y presentarse, manteniendo contacto visual, llamándole por su nombre y recibiéndole con amabilidad.

Para validar se requiere dar valor o legitimar, con gestos y/o palabras, la perspectiva de la otra persona, sin que necesariamente estemos de acuerdo. Si se acepta y legitima lo que alguien nos dice, porque tiene sus razones para verlo y sentirlo de manera diferente a cómo lo percibimos nosotros, tendremos mayor probabilidad de que nuestras perspectivas y opiniones sean escuchadas y tenidas en cuenta. Sin embargo, cuando juzgamos o invalidamos cuanto nos comunican, habrá mayor probabilidad de que se corte el proceso interactivo.

Validar es abrir la puerta al encuentro. Es reconocer la perspectiva de nuestro interlocutor, aceptarla, comprenderla y respetarla. Es decir, dar valor a su punto de vista, legitimarlo, sin perder la perspectiva profesional.

\section{Habilidades de comunicación que nos permiten validar}

Son muchas las habilidades que permiten validar, se podría decir que todas aquellas que facilitan la comunicación. Necesitamos esencialmente: a) Escuchar activamente cuál es su perspectiva; b) Empatizar con ella; c) Hablar en Mensaje "yo" lo que significa expresar y responsabilizarse de la propia perspectiva, Ej. "En mi opinión..." "Mi impresión es..." "Yo opino que... ¿Qué opinas tú?" 
Tabla 3. Cinco reglas básicas para validar y reducir resistencias.

\begin{tabular}{|c|c|}
\hline Reglas validación & Ejemplos \\
\hline $\begin{array}{l}\text { 1. Escuchar sin juzgar: dejar al } \\
\text { lado los prejuicios, respetar la } \\
\text { perspectiva del otro }\end{array}$ & $\begin{array}{l}\text { - Qué difícil debe ser... } \\
\text { - Qué duro... }\end{array}$ \\
\hline - 2. Verbalizarlo & $\begin{array}{l}\text { - "Estoy escuchándole y me da la impresión que } \\
\text { algo le preocupa" }\end{array}$ \\
\hline $\begin{array}{l}\text { - 3. No bloquear la emoción: } \\
\text { normalizarla }\end{array}$ & $\begin{array}{l}\text { - Ante una persona que expresa: “iEstoy } \\
\text { agotado...!" } \\
\text { - No ayuda decir: “Cálmese”" o "No se } \\
\text { preocupe... Anímese!!” } \\
\text { - Si ayuda: Es normal estar cansado después de } \\
\text { tantos tratamientos. }\end{array}$ \\
\hline $\begin{array}{l}\text { 4. Resumen de lo escuchado, } \\
\text { devolver información recibida, } \\
\text { parafrasear }\end{array}$ & $\begin{array}{l}\text { - Así es que lo que me está queriendo decir es } \\
\text { que... }\end{array}$ \\
\hline $\begin{array}{l}\text { 5. Silencio como acompañamiento } \\
\text { empático. Importancia del lenguaje } \\
\text { no verbal }\end{array}$ & \\
\hline
\end{tabular}

Adaptado de: Costa y Arranz ${ }^{(5)}$.

\section{$3^{\circ}$. Exponer mi perspectiva profesional: Informar y precisar para orientar.}

Ofrecer información desde lo que a cada estamento sanitario le incumbe, disminuye la incertidumbre y aumenta la percepción de control. Se trata de informar con honestidad, claridad y sencillez al ritmo del paciente. Adaptar el lenguaje a su contexto, así como realizar esquemas y dibujos son de ayuda para transmitir la información y facilitar el diálogo. También es importante tener en cuenta que el paciente tiene derecho a saber, y a no saber si no quiere. En la información que da el profesional sanitario hay dos dimensiones relevantes: a) facilitar la información clínica objetiva sobre el problema: "La leucemia es..." y b) transmitir la opinión técnica sobre mismo: "Para tratar su enfermedad, en estos momentos tenemos varias opciones. Por eso le aplicamos este tratamiento que le comentó el médico..."

$4^{\circ}$. Explorar la perspectiva del paciente: Preguntar para saber.

Nadie se suele comprometer a nada que perciba como impuesto. Joaquín, es psicooncólogo y esta mañana se ha encontrado con Carmen, una paciente que ha acompañado desde su diagnóstico de leucemia. Se sentía muy nerviosa.

Ps: Hola Carmen ¿Hay algo que te preocupe especialmente en estos momentos?

Pac: Nada, soy muy bruta, no paro de meterme con mi hermana, que ha venido a cuidarme. 
Ps: No está siendo fácil parece...cuando una persona tan amable como tú se mete con su hermana, probablemente se siente muy angustiada por algo...

Pac: La verdad es que temo que me queda poco

Ps: ¿Qué te hace pensar eso?

Pac: Que ya no tengo tratamiento, sólo me ponen sangre o plaquetas.

Ps: ¿Quieres hablar con tu médico sobre ello?

Pac: Si, me ayudaría mucho

Ps: Tienes mucha fortaleza. Afrontar esta situación no es nada fácil...

Hay herramientas que resultan especialmente relevantes para evitar malos entendidos, corregir errores y omitir imposiciones al facilitar al interlocutor la opción de elegir. Así, por ejemplo, antes de lanzarse a informar de un modo exhaustivo, podría preguntarse: qué es lo que sabe y qué le gustaría saber, sobre la situación particular que está viviendo el paciente. Puede ser un diagnóstico reciente o un nuevo tratamiento. Si se opta por preguntar, la información resultará más significativa para el paciente al basarse en lo que este sabe y se corregirán mejor los mitos, errores y expectativas desajustadas que pudiera tener.

Explorar la perspectiva del paciente o de otros interlocutores (familiares, profesionales del equipo...) conlleva preguntar sobre lo que les preocupa (¿Qué es lo que más le preocupa?) y sobre las alternativas de solución a sus preocupaciones y problemas (¿Qué es lo que más le ayudaría?). La pregunta abierta focalizada es una habilidad clave para no presuponer lo que piensan los demás, para promover el compromiso y la opción a elegir. Permite, así mismo, evitar las imposiciones, identificar si estamos interpretando en función de nuestra historia biográfica y facilitar la adhesión al tratamiento ${ }^{(30)}$. En suma, conseguir que el interlocutor reflexione y decida por sí mismo, en la medida de lo posible.

Hay dos tipos de preguntas: abiertas ("¿Qué le sucede? ¿En qué puedo ayudarle?”) y cerradas (“iLe duele aquí, ¿verdad?"). Estamos proponiendo realizar preguntas abiertas y focalizadas hacia un objetivo. Las preguntas contribuyen también a potenciar los recursos que todas las personas tienen para afrontar las adversidades y reducir resistencias ${ }^{30}$. Es tan importante que merece incorporarse en el abanico habitual del clínico en su relación cotidiana con los pacientes y familiares. Así, por ejemplo, en vez de decir: "María tiene que poner de su parte y caminar un poco", podría decirse "María, a partir de ahora, ¿qué le ayudaría a ponerse de pie y caminar un poco cada dia?", " ¿hay alguna cosa de la que quiere que hablemos?”. Preguntar antes de comentar directamente nuestra perspectiva ayuda a abordar el siguiente punto: llegar a acuerdos en la toma de decisiones que afectan al paciente. Necesitamos que se haga cargo de su situación.

\section{$5^{\circ}$. Acordar la intervención: toma de decisiones compartida (TDC). Dialogar para encontrarse.}

De la comunicación a la deliberación moral: Laura viene a la consulta del psicooncólogo para plantear la percepción que tiene la familia de la situación que atraviesa su hermana, que padece un síndrome de Ewing en proceso final de vida.

L: Tenemos la impresión de que mi hermana está aguantando lo que no está en los escritos, para no darnos el disgusto de que no ha luchado lo suficiente por mantenerse viva. No podemos seguir viéndola sufrir así. 
Ps: Entiendo vuestra preocupación, es un tema delicado en el que es importante involucrar al médico que la lleva. Para ello nos gustaría hacer una reunión con vosotros y, posteriormente, hablar con ella y su marido. Es importante que podáis expresarle lo que sentís y que, juntos pudiéramos ver las opciones que hay y tomar la decisión que considere más oportuna. Os acompañaremos en todo este proceso, ¿cómo lo veis?

La aplicación de los todos los medios técnicos disponibles puede ir acompañada de consecuencias no deseadas por los pacientes y ser éticamente inadmisibles. Estos escenarios suelen darse con mayor frecuencia en pediatría y en los ancianos ${ }^{(28)}$. Ello conduce a la necesidad de una toma de decisiones compartida con la familia que permita la adecuación del esfuerzo terapéutico y con el niño adecuando la información a su madurez de comprensión y competencia. A pesar de las dificultades, como la falta de tiempo, la presión asistencial y/o las resistencias al cambio, se propone implicar a los pacientes y sus familiares, respetando su historia de valores para alcanzar sus objetivos de salud.

En este proceso interactivo, el profesional sanitario valora la capacidad de toma de decisiones del paciente o representantes y aporta sus conocimientos, así como información de los riesgos y beneficios en referencia a una intervención diagnóstica o terapéutica. El paciente y/o su familia por su parte, expresa sus preferencias y preocupaciones respecto a su experiencia con el problema de salud, así como sus historias de valores ${ }^{(31)}$.

\section{Conclusiones}

La confianza y la conexión con el paciente es la base de la relación clínica. El arte de generar confianza en una consulta tiene sus cimientos en la empatía y la validación. Cuando un paciente se siente escuchado, entendido y atendido, se abre al profesional y facilita la intervención ${ }^{(33-34)}$.

El perfil sanitario actual, cada vez más especializado, puede conducir a minimizar el componente comunicativo-relacional incuestionable. Como destaca el editorial publicado hace ya más de 14 años en The Lancet ${ }^{(34)}$ : "El énfasis del adiestramiento sanitario actual se pone en las ciencias duras: anatomía, fisiología, bioquímica, patología, microbiología y farmacología, pero en la práctica clínica los profesionales tienen que tratar de comprender a los pacientes, sus historias y peculiaridades, para poder proporcionarles el mejor cuidado posible. En este lado 'blando' de la Medicina las ciencias 'duras' les son de escasa ayuda". Ya ningún científico puede considerar la dimensión psicoemocional como ciencias "blandas". Se pone de manifiesto la necesidad del aspecto comunicativo afectivo y asertivo entre el paciente y el médico para adquirir una auténtica competencia clínica ${ }^{(27)}$.

El protocolo básico propuesto permite establecer, de un modo sistemático e integral, un encuentro auténtico con el paciente, lo que genera satisfacción para el profesional y motivación para el paciente de comprometerse en su proceso saludenfermedad. 


\section{Agradecimientos}

Quisiéramos agradecer a tantos pacientes y sus familias por su inestimable ayuda. Así mismo a Ramón Bayés, Pilar Barreto, Javier Barbero y Hernán Cancio, por su participación en la elaboración del protocolo. A las personas que configuraron un verdadero equipo interdisciplinar en el Servicio de Hematología del Hospital Universitario La Paz, liderado por Fernando Hernández Navarro, Jesús García de Bustos y la supervisora María Moreno, cuando era una utopía el trabajo en equipo. Al innovador equipo de psicólogos del Instituto Antae que difunden compasión, acogida y presencia en el mundo sanitario, para favorecer un contexto de encuentros y transcender tanto sufrimiento. Pero sobre todo, a los pacientes, nuestros sabios preferidos, con los que hemos tenido el privilegio de compartir "gozos y sombras" y que tanto continúan enseñándonos.

\section{Referencias bibliográficas}

1. Caballero Martínez F. Simulación: el entorno clínico virtual. Educ Med 2017; 18: (Supl 1), 12-9.

2. Simpson M, Buckman R, Stewart M, Maguire P, Lipkin M, Novack D, Till J. Doctorpatient communication. BMJ 1991; 303: 1385-7. [Acceso 17 de octubre 2019]. Disponible en https://www.ncbi.nlm.nih.gov/pmc/articles/PMC1671610/ doi: 10.1136/ bmj.303.6814.1385

3. Bimbela JL. Cuidando al profesional de la salud. Habilidades emocionales y de comunicación. Granada: Escuela Andaluza de Salud Pública; 2005.

4. Ruiz R, Caballero F, García de Leonardo C, Monge D, Cañas F, Castaño P. Enseñar y aprender habilidades de comunicación Clínica en la Facultad de Medicina. La experiencia de la U Francisco de Vitoria (Madrid). Educ Med 2017; 18:289-97. doi: 10.1016/j. edumed.2017.03.026

5. Costa M, Arranz P. Comunicación y counselling en Cruzado JA, editor. Manual de Psicooncología. Madrid: Pirámide; 2013. p. 55-78

6. Di Blasi Z, Harkness E, Ernst E, Georgiou A, Kleijnen J. Influence of context effects on health outcomes: A systematic review. Lancet 2001; 357:757-62.

7. Blech J. El destino no está escrito en los genes. Barcelona: Destino; 2012.

8. Buckman R. Communication and emotions. BMJ 2002; 28: 697-700. doi: 10.1136/ bmj.325.7366.672

9. Zubieta JK, Stohler CS. Neurobiological mechanisms of placebo responses. Ann N Y Acad Sci 2009; 1156: 198-210. doi: 10.1111/j.1749-6632.2009.04424.x

10. López E, Costa M. Dar sentido a la vida cuando la incomunicación nos la complica. Madrid: Pirámide; 2018.

11. Gómez M. Los valores de la medicina en el s XXI. Organización Médica Colegial, 2008 [Acceso 17 de octubre 2019]. Disponible en: http://www.medicosypacientes.com/ articulo/los-valores-de-la-medicina-en-el-siglo-xxi

12. Henry SG, Holmboe ES, Frankel, RM. Evidence-based competencies for improving communication skills in graduate medical education: A review with suggestions for implementation. Med Teach 2013; 35: 395-403. doi: 10.3109/0142159X.2013.769677

13. Ley 41/2002, de 14 de noviembre, básica reguladora de la autonomía del paciente y de derechos y obligaciones en materia de información y documentación clínica. [Acceso 17 
de octubre 2019]. Disponible en: https://www.boe.es/buscar/pdf/2002/BOE-A-200222188-consolidado.pdf

14. Carta de los Derechos del Paciente aprobado por la Asociación Americana de Hospitales el 6 de febrero de 1973. Texto publicado con la autorización de la Asociación Americana de Hospitales y traducido de la redacción del Boletín de la Oficina Sanitaria Panamericana a partir del original. [Acceso 17 de octubre 2019] Disponible en: http://legisalud.gov.ar/ dels/declaracion_derechos_paciente.pdf

15. García Huete E. (Dir). Counselling en VIH/SIDA. Guía de actuación para profesionales sociosanitarios. [Acceso 17 de octubre 2019]. Disponible en: https://docplayer. es/17331936-Counselling-en-vih-sida.html

16. Arranz P, Cancio H. Counselling: habilidades de información y comunicación con el paciente oncológico. En: Gil F, editor. Manual de Psico-oncología. Madrid: Nova Sidonia; 2000. p. 39-56.

17. Bayés R. ¿Qué puede ofrecer un viejo profesor a las nuevas generaciones? Health, Aging End of Life 2016; 1: 9-15.

18. Sinclair S, Torres, MB, Raffin-Bouchal S, Hack TF, McClement S, Hagen NA, Chochinov HM. Compassion in health care: an empirical model. J Pain Symptom Manage 2016; 51 : 193-203. doi: 10.1186/s12909-013-0695-0

19. Kurtz S, Silverman J, Benson J y Draper J. Marrying content and process in clinical method. teaching: enhancing the Calgary-Cambridge guides. Acad Medicine 2003; 78 : 802-9. doi:10.1097/00001888-200308000-00011

20. Arranz P, Bayés R. Tratamiento de Soporte (VI) Apoyo Psicológico. En: González Barón M, Ordóñez A, Feliú J, Zamora P, Espinosa E, Castro J, editores. Oncología Clínica. 1. Fundamentos y Patología General (2 ${ }^{\mathrm{a}}$ ed) Madrid: McGraw- Hill- Panamericana; 1997. p. 649-57.

21. Arranz P, Salinas A. Comunicación: el arte de hacer preguntas. En: E López Imedio. Enfermería en cuidados paliativos. Madrid: McGraw Hill Panamericana; 1998: 221-6.

22. Arranz P, Costa M, Bayes R, Cancio H, Magallón M, Hernandez F. Emotional support in Haemophilia. Canada: WFH; 1999.

23. Arranz P. La comunicación en cuidados paliativos. Med Pal 2001, 8:F1, 26-31.

24. Arranz P, Barbero J, Barreto P, Bayes R. Intervención emocional en cuidados paliativos Modelo y protocolos. Barcelona: Ariel Ciencias Médicas; 2003.

25. Benito E, Arranz P, Cancio H. Herramientas para el autocuidado del profesional que atienden a personas que sufren. Form Med Contin 2011; 18: 59-65.

26. Costa M, Arranz P. Comunicación y Counselling en oncología. El encuentro con el paciente oncológico. En: Cruzado JA, editor. Manual de Psicooncología, Madrid: Pirámide; 2013. p. 55-77.

27. García-Llana H y Rodríguez Rey R. El acompañamiento a los profesionales que trabajan con las personas que se encuentran en el proceso de morir y con sus familias. Revista de Treball Social (RTS). Col-legi Oficial de Treball Social de Catalunya; 2017; 210:115-27.

28. Coca C, Rodríguez Rey R, Arranz P. Claves de comunicación con pacientes, familiares y equipo asistencial en Nefrología. En: Alonso Babarro A, García-Llana H, Leiva Santos JP, Sánchez Hernández R, editores. Manual de Cuidados Paliativos Renales. Madrid: Pulso Editorial; 2018.p.107-26.

29. Patterson K, Grenny J, McMillan R, Switzler A. Conversaciones cruciales. Barcelona: Urano; 2016.

30. Costa M, López E. Manual para la ayuda psicológica. Dar poder para vivir. Más allá del Counselling. Madrid: Pirámide; 2006. 
31. Soler J, Raurich J. Comunicación y atención primaria para alumnos de medicina. Lleida: Universidad de Lleida; 2015.

32. Barbero J, Prados C, González A. Hacia un modelo de toma de decisiones compartidas para el final de la vida. Psicooncología 2011; 8:143-68. doi: 10.5209/rev_PSIC.2011. v8.n1.11

33. Benedetti F. Placebo and the new physiology of the doctor-patient relationship. Physiol Rev 2013; 93: 1207-43. doi: 10.1152/physrev.00043.2012

34. Fallowfield L, Jenkins V. Communicating sad, bad, and difficult news in medicine. Lancet. 2004 Jan 24; 363(9405): 312-9. doi: 10.1016/S0140-6736(03)15392-5

35. Pinto H. Comentarios al artículo Enseñar y aprender habilidades de comunicación clínica en la Facultad de Medicina”. La experiencia del Francisco de Vitoria (Madrid). Carta al Director. Edu Med 2019; 20(3). 
